\title{
Developing Metacognitive Models for Team-Based Dynamic Environment Using Fuzzy Cognitive Mapping
}

\author{
Jung Hyup Kim, Gretchen A. Macht, Ling Rothrock, and David A. Nembhard \\ Department of Industrial and Manufacturing Engineering, \\ Pennsylvania State University, USA \\ \{jzk170, gam201, Irothroc, dan12\} @psu.edu
}

\begin{abstract}
In this paper, by using Fuzzy Cognitive Mapping (FCM) technique, we developed the metacognitive models for team-based dynamic environment. Preliminary findings from our metacognitive studies provided a possible metacognitive framework in dynamic control tasks $[1,2]$. By analyzing metacognition, performance, and communication data between team, we are able to develop the team-based evolving metacognitive models for the dynamic environments using a fuzzy cognitive map. In this research, a human-in-the-loop simulation experiment was conducted to collect communication data, objective performance data (operator on-time action performance), and subjective rating data (retrospective confident metacognitive judgment) from 6 dyads (12 participants). Within the Anti-Air Warfare Coordinator (AAWC) simulation domain, the simulation test bed provides an interactive simulating condition in which the monitoring team must communicate with their team member to defend their ship against hostile aircraft.
\end{abstract}

Keywords: Metacognition, Team Performance, Human-in-the-loop simulation, Fuzzy Cognitive Map.

\section{Introduction}

The need to develop more advanced on-the-job training methods has become a growing concern in many industries because people not only work as individuals, but as members of teams. Although advanced technology provides the ability to develop more effective training approaches to novice workers, building effective team training methods is still on-going research area. To address this critical need, this research investigated the different behavior models based on metacognition and communication between team members using Fuzzy Cognitive Mapping (FCM) techniques. FCM is a "mental landscape" of the elements (e.g. actors, values, goals, and trends) in a fuzzy feedback system. FCM can demonstrate the links between causal events of dynamic tasks and human behavior with the change of time. The map lists the fuzzy rules related with events to show causal flow paths like Hasse diagram [3]. The FCM enables job trainers to evaluate trainees' internal learning states, and to help the instructors to choose several possible actions. In this research, we developed a humanin-the-loop simulation, which emulates a computer-based dynamic control task to 
identify navigational cognitive behavior in order to collect metacognition and communication data in a team-based training environment. The domain of this simulation test bed is AAWC (Anti Air warfare Coordinator). This is an interactive simulation in which a controller must defend his/her ship against hostile aircraft. Data from the experiment indicated that metacognitive monitoring behaviors which were measured using subjective rating methods showed different patterns between teammates. Role A (executing appropriate actions based on Rules of Engagements (RoE)) showed a significant positive correlation between the metacognition and performance ${ }^{1}$ of identification rules while Role B (controlling Defense Counter Aircrafts (DCA) to update the information of unknown hostile aircrafts) was not (Role A: $r=0.474, p<0.05$; Role B: $r=-0.28, p=0.184$ ). Since teammates communicated with each other only through the telephone, Role A had to obtain and understand information of unknown hostile aircrafts through the communication with Role B, and this contributed to different metacognitive judgments between teammates [1]. It has been suggested that the key to success in teamwork lies to a great extent in achieving not only successful team cooperation, but also efficient team communication [4]. In this paper, by using FCM techniques and data (metacognition and communication) from the previous teambased AAWC experiment, we develop the team-based metacognitive mapping models in a dynamic control tasks. This work provides understanding on how the communication and metacognitive behavior influence team members.

\section{Literature Review}

\subsection{Metacognition}

Metacognition is mainly the mind's ability to monitor and control human cognition. According to the Nelson and Narens's metacognitive model [5], human metacognition is significantly influenced by information flows between "meta-level" and "objectlevel". The object-level consists of ongoing cognitive processes, such as perception, problem solving, and learning. The meta-level is about a persons' own understanding related to his or her object-level cognition. The information flows between these levels are unidirectional. The information channel from object-level to meta-level is called metacognitive monitoring, while the other channel from meta-level to objectlevel is called metacognitive controlling. The data entity of the information flows between these levels consists of metacognitive knowledge or meta-memory. The meta-memory is a self-awareness of memory which can reflect how people learn and use their memories. In other words, it is the awareness of one's own cognition. These meta-memories can be transferred to meta-level through the metacognitive monitoring. It is the ability to make accurate accessing the current state of cognitive activities on object-level. By using metacognitive monitoring process, people will produce more effective regulation to improve their learning [6]. After that, the metacognitive control can regulate ongoing cognitive activities such as a decision-making procedure

\footnotetext{
${ }^{1}$ Correct response rate for identifying hostile aircrafts: number of correct responses/ total number of identification tasks for unknown hostile aircrafts.
} 
for using new tactics to solve a difficult problem. In this paper, we focus on the ability to make accurate monitoring judgments for the current state of cognitive activities because accurate monitoring of learning is one of the critical elements in metacognition [7].

\subsection{Fuzzy Cognitive Mapping (FCM)}

Fuzzy cognitive mapping (FCM) was developed by Kosko in 1986. FCM is an extension of conventional cognitive maps of binary logic set theory. Cognitive maps (CMs) are defined as a type of mental processing composed of a series of modeling decision making in individual mind maps and social political systems. CMs have been studied in various fields due to a strong visual representation of causal relationships and a clear comparison of mental models to reality. However, conventional cognitive maps make knowledge acquisition oppressively burdensome from insufficient decision information, different experts. Hence, a need to represent causal relationships of linguistic quantities becomes very important. FCM can express various degrees of increase or decrease of casual relationships. It is a very powerful tool to represent and compute the "strength of impact" of causal flow paths in dynamic environment. Fuzzy set theory is behind the computational theory of FCM. Since Lotfi Zadeh published a paper titled "Fuzzy Sets" [8], the various applications using fuzzy sets have been successfully tested in the control engineering distributed networks [9], health care [10], decision support systems [11], and situation awareness for army infantry [12].

\section{Method}

Our current work focuses on developing the representation of metacognitive mapping model in team-based dynamic control tasks by using FCM technique.

\subsection{Dynamic Decision-Making Task Human-In-The-Loop Simulation}

The AAWC (Anti Air warfare Coordinator) test bed is interactive simulation in which a controller must defend his/her ship against hostile aircraft.

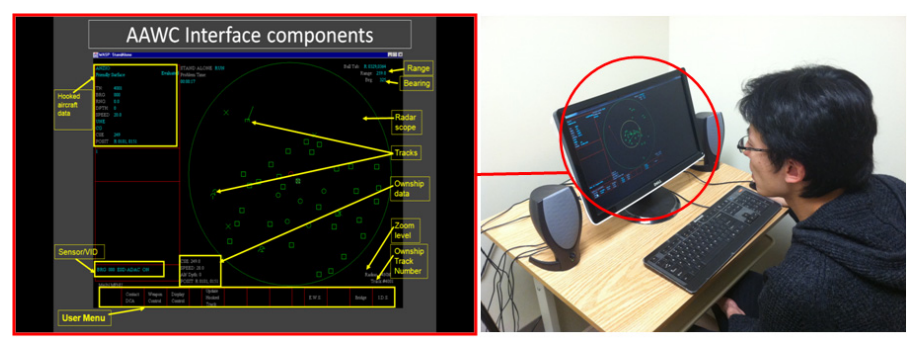

Fig. 1. AAWC interface (left) and experimental setup (right) 
A total of 12 male engineering graduate students (age 18 or older) participated with little to no previously established relationships. The experiment was single gender to avoid potential gender interactions in the current study. Participants were voluntary and screened for prior experience with the task domain. The experiment consists of two sessions - a training session and an experiment session. The participants participated in an initial training session (Day 1), which lasted 60 minutes. During this session the participants were trained on the specific skills and provided with feedback on their task. Participants gained experience and understanding of the tasks based on the feedback provided by the instructor. The practice scenario lasted approximately five minutes with a task complexity that was easier than the actual experiment. Based on the result of the pilot test, participants were ready to engage in the actual experiment after they executed the practice simulation for the third time. The participants after being trained underwent an experiment session (Day 2), which lasted approximately 90 minutes. During this session the participants were required to perform certain tasks based on the scenario. In the experiment, four scenarios are developed and events in each scenario have their specific sequence to occur. Each scenario was designed to run 15 minutes long. Freeze occurred randomly between 10 and 15 minutes after the start of the scenario. Once the simulation was frozen, participants required answering retrospective confidence judgment (metacognition) probes. Retrospective confidence judgment comes from metacognitive monitoring processes associated more directly with retrieval [13]. The probes used for measurement are shown below:

- How well do you think you have detected the objects in your airspace?

- How well do you think you are aware of the current overall situation of your airspace?

- How well do you think you are aware of where the overall situation of your airspace is heading?

\subsection{Team Selection}

Team assignments were determined based on the Big Five Inventory (BFI) as the specific measurement tool for FFM personality conducted on the first of the two days of the study [14]. FFM extraversion formed the basis for dyad membership, wherein dyads were either two introverts (II), or one introvert and one extravert (EI). Each dyad had at least one introvert, also as a part of variance reduction, and that introvert was always assigned to the second of the two task roles (DCA). To construct these dyads, Ward's clustering [15] was used as an iterative pairwise comparison of Euclidean based distances, in order to group participants with similar extravert scores. The remaining introverts were then added to the heterogeneous EI groups, wherein assignments were optimized based on the maximum variance between the levels of extraversion-introversion, while minimizing the variation among the remaining four FFM factors. The result was three distinct teams in each category of dyad. The placement of the extraverts within roles in the dyads did not convey any particular status to either individual within the dyad (e.g., [16, 17]). Both the extrovert or the introvert were responsible to take appropriate actions based on the Rules of Engagement (RoE); where the introverted individual always remained responsible to control 
Defense Counter Aircrafts (DCA). This team-based environment provides the condition in which the monitoring team (Roles A and B) must communicate with their team member to defend their ship against hostile aircraft.

\subsection{Developing Augmented FCM}

By using the communication and performance data between teammates, we defined eleven important behavior concepts (see Section 3.4) that directly relate to the task at hand. Simple FCMs which have bivalent nodes and edges can be addressed based on communication data. Causal edges take values in $\{-1,0,1\}$. These simple FCMs additively combine to form new FCMs [3]. The $W_{\mathrm{i}}$ are positive or negative weights for the $i^{\text {th }}$ FCM $F_{i}$. The weights state the relative value of each FCM in the environment. In this paper, we assume that the weights of any sub-graph of the FCMs are equal and take them as unity: $W_{i}=1$. Figure 2 shows an example of the augmented FCM for identification task. $F_{R O E}$ and $F_{D C A}$ are simple FCMs for $i^{\text {th }}$ identification tasks in dynamic environment $(\mathrm{i}=1 \ldots \mathrm{n} ; \mathrm{n}=$ total number of identification tasks).

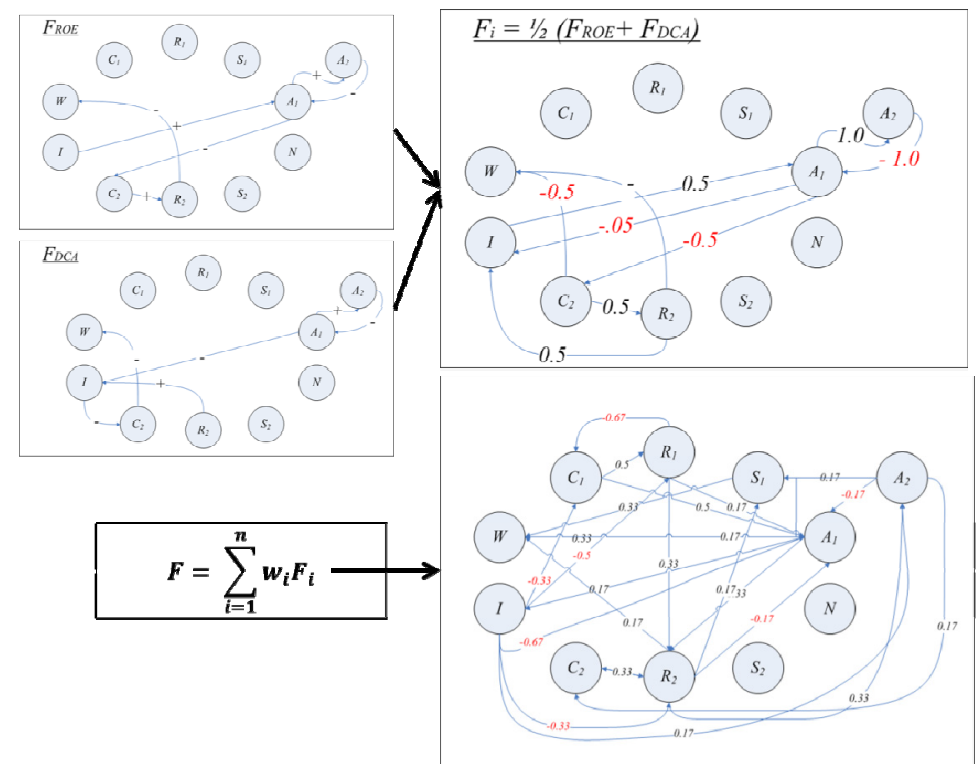

Fig. 2. Procedure for developing Augmented FCM

\subsection{Performance Metrics}

In this experiment, we collected operator on-time action performance, retrospective metacognitive judgment, and communication data between teammates.

Operator On-Time Identification Performance (OIP): It is defined as the degree of on-time correct action of a dynamic control task. A time window is a construct that specifies the availability of an action opportunity which leads to the required situation 
[18]. In our experiment, the main goal is correctly identifying every unknown hostile aircrafts at appropriate times. Here, we only consider hostiles or assumed hostiles. Operator on-time Action Performance is simply calculated by:

$$
\mathrm{OIP}=\mathrm{CR} \times 100 / \mathrm{TW} \_ \text {id }
$$

Where:

- OIP: operator on-time identification performance

- CR: number of correct identification responses

- TW_id: total number of time window assigned for unknown identification task

Retrospective Confidence Judgment (RCJ): Participants state their confidence level for their responses before knowing whether they are correct or incorrect. Confidence level ratings are compared to the accuracy of past retrieval. We collected self-rating scores (scale: 1 to 100) during the testing sessions.

Communication Data: The participants for the two roles were kept in separate rooms, thereby eliminating gestural non-verbal communication and other sight related cues. Therefore Role A had to obtain and understand information of unknown hostile aircrafts through verbal communication with Role B. Although, the two monitors are synchronized, no direct communication was available through the computer. Communication metrics were the number of utterances (e.g. Table 1 has 7 utterances; 4 RoE and 3 DCA), word count (e.g. 10 words in the first utterance in Table 1), and duration ([19-21]). For each of the 24 dyad and trial combinations, the audio was transcribed, timed, with words, utterances, and durations determined by two raters. The analysis on communication data between team members provided valuable insight on how to effectively support human cognition within the decision-making process. Based on the communication and performance data between teammates, we developed eleven important behavior concepts that directly relate to the task at hand (see Table 2).

Table 1. Example of Role Dialogue to Complete ID Tasks

\begin{tabular}{c|l|l}
\hline Role & \multicolumn{1}{|c}{ Dialogue } & \multicolumn{1}{|c}{ Request Type } \\
\hline ROE: & Unknown aircraft number 17, bearing 106, range 21.6 nautical miles. & Call ID $\left(\mathrm{C}_{1}\right)$ \\
DCA: & Yes. I'll send something towards it. & Response ID $\left(\mathrm{R}_{1}\right)$ \\
ROE: & Anything on number 17? & Re-Call ID $\left(\mathrm{C}_{2}\right)$ \\
DCA: & Target 17 is a hostile strike aircraft. & Identification $(\mathrm{I})$ \\
ROE: & Got it. Thanks. & $\operatorname{Re}-$ Affirm ID $\left(\mathrm{A}_{2}\right)$ \\
ROE: & It did not respond to my warnings, so I shot it down. & Shot $\left(\mathrm{S}_{1}\right)$ \\
DCA: & Got it. Thanks. & Response Shot $\left(\mathrm{S}_{2}\right)$ \\
\hline
\end{tabular}

Table 2. Description of Behavior Concept Nodes

\begin{tabular}{l|l}
\hline \multicolumn{1}{c|}{ Behavior Concepts } & \multicolumn{1}{c}{ Description } \\
\hline Call ID $\left(\mathrm{C}_{1}\right)$ & Ask for identification of an Unidentified track Number \\
Response ID $\left(\mathrm{R}_{1}\right)$ & Recognizing the Call ID \\
Affirm ID $\left(\mathrm{A}_{1}\right)$ & Confirming the ID \\
\hline
\end{tabular}


Table 2. (Continued)

\begin{tabular}{l|l}
\hline Re-Call ID $\left(C_{2}\right)$ & Re-asking for the identification of a unknown aircraft \\
\hline Re-Response ID $\left(\mathrm{R}_{2}\right)$ & Recognizing the Re-Call ID \\
Re-Affirm ID $\left(\mathrm{A}_{2}\right)$ & Re-conforming the ID \\
Shot $\left(\mathrm{S}_{1}\right)$ & Shooting of the Aircraft which poses a potential threat to the ship \\
Response Shot $\left(\mathrm{S}_{2}\right)$ & Confirming the Shot \\
Wait $(\mathrm{W})$ & Searching the Unidentified or Hostile or Assumed Hostile Aircraft \\
Identification (I) & Identification action of selected unknown aircraft \\
Noise (N) & Conversation that have nothing to deal with the task at hand \\
\hline
\end{tabular}

FCM inferences: After the FCMs were delineated, we could determine the system's steady state by using the auto-associative neural network methods [22]. This method only considers outcomes and dynamics of each node. Following this method, the value of each node $\left(\mathrm{C}_{\mathrm{i}}\right)$ in iteration $(\mathrm{t})$ can be computed as:

$$
C_{i}^{t}=f\left(\sum_{j-1}^{n} C_{j}^{t-1} W_{j i}+C_{i}^{t-1}\right)
$$

Where:

$-C_{i}^{t}$ : Values of the node $\mathrm{i}$ at the end of the iteration.

$-C_{j}^{t}:$ Values of the subsequent node $\mathrm{j}$ at the beginning of the iteration

$-W_{j k}$ : Corresponding strength of the link from node $\mathrm{j}$ to node $\mathrm{i}$

$-f$ : Threshold function that transforms the result of the multiplication. Usually the logistic function that assumes the form $1 /\left(1+e^{-x}\right)$ is used.

\section{$4 \quad$ Results}

\subsection{Descriptive Statistics}

EI group's OIP performance mean is higher than II group (see Table 3), but there is no significant performance difference between these groups based on the result of ANOVA test $(F=0.68, p=0.418)$. In addition, RCJ means show that there is no significant different between EI and II group $(F=0.00, p=0.977)$.

Table 3. Metacogntion and Task Performance results

\begin{tabular}{c|ccccccc}
\hline Team & Variable & $N$ & Mean & StDev & Minimum & Median & Maximum \\
\hline \multirow{2}{*}{ EI } & RCJ & 12 & 66.00 & 5.7 & 40 & 65 & 86.75 \\
& OIP & 12 & 45.84 & 19.82 & 28.6 & 42.9 & 85.7 \\
\hline \multirow{2}{*}{ II } & RCJ & 12 & 66.25 & 22.27 & 20 & 70 & 90 \\
& OIP & 12 & 40.48 & 10.71 & 21.4 & 42.9 & 50 \\
\hline
\end{tabular}

\subsection{Team-Based Metacognitive Models Using Fuzzy Cognitive Mapping (TBM-FCM) in AAWC System}

After combining team communication and metacognition data, we are able to construct FCM matrices for each team using the nodes in Table 1. It allows developing 
different cognitive map patterns between groups by including the union of the causal concepts of the system. Figure 3 and 4 show examples of the augmented TBM-FCM for both EI and II group.

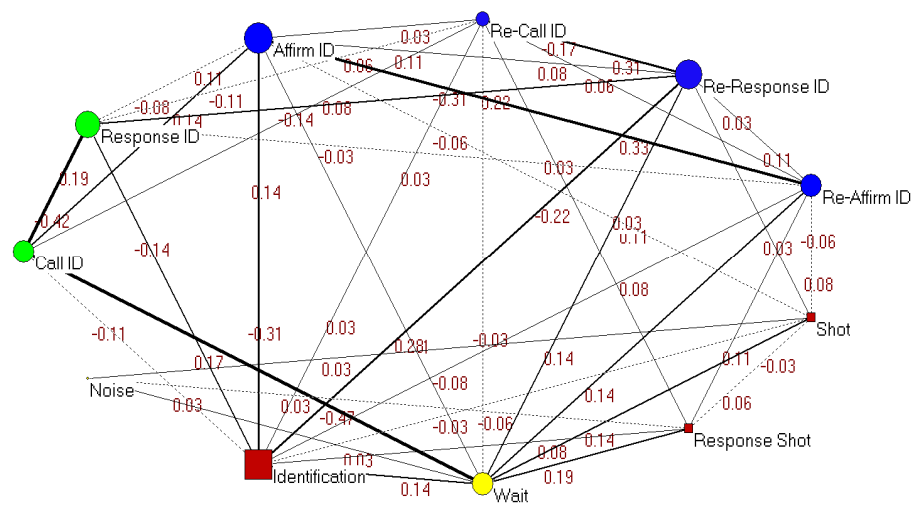

Fig. 3. TBM-FCM for EI Group

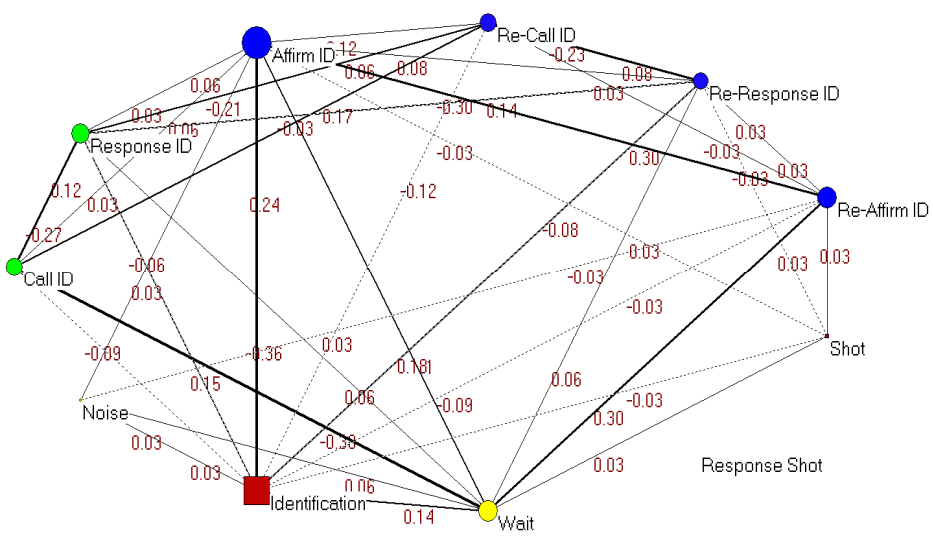

Fig. 4. TBM-FCM for II Group

\subsection{Simulation of Steady State for the FCMs}

According to the task performance and metacognition data, both groups show either Over-Confident $\left(\mathrm{RCJ}_{\mathrm{j}}>\mathrm{OIP}\right)^{*}$ or Under-Confident $\left(\mathrm{RCJ}_{\mathrm{j}}<\mathrm{OIP}_{\mathrm{j}}\right)^{*}$ metacognitive behavior during the experiment. Moreover, the communication data reveals that Noise node can cause the misunderstanding between teammates. Hence, we designed two conditions for both metacognition (Over and Under-Confident) and communication status ("Clean" and "Noise") in each group. Based on our experimental data, we are

* $\mathrm{j}=1, . . \mathrm{m} ; \mathrm{m}=$ number of dyad and trial combinations. 
able to generate three different cases for each group and simulate them until the system reach to the steady state (see Table 4) using equation 2 . The values show the effect of communication status and metacognition in the team performance.

Table 4. Steady State Results for the FCMs

\begin{tabular}{|c|c|c|c|c|c|c|}
\hline & & $E I$ & & & II & \\
\hline Communication & Noise & Clean & Noise & Noise & Clean & Noise \\
\hline Metacognition & $\begin{array}{c}\text { Over } \\
\text { Confident }\end{array}$ & $\begin{array}{c}\text { Over } \\
\text { Confident }\end{array}$ & $\begin{array}{c}\text { Under } \\
\text { Confident }\end{array}$ & $\begin{array}{c}\text { Over } \\
\text { Confident }\end{array}$ & $\begin{array}{c}\text { Over } \\
\text { Confident }\end{array}$ & $\begin{array}{c}\text { Under } \\
\text { Confident }\end{array}$ \\
\hline $\mathrm{C}_{1}$ & 0.41 & 0.38 & 0.37 & 0.44 & 0.40 & 0.42 \\
\hline $\mathrm{R}_{1}$ & 0.455038 & 0.482606 & 0.515562 & 0.533515 & 0.514912 & 0.5 \\
\hline $\mathrm{A}_{1}$ & 0.556693 & 0.433616 & 0.548736 & 0.536218 & 0.534958 & 0.576646 \\
\hline $\mathrm{C}_{2}$ & 0.509092 & 0.571576 & 0.509636 & 0.475688 & 0.472333 & 0.468993 \\
\hline $\mathrm{R}_{2}$ & 0.541461 & 0.580115 & 0.529245 & 0.496961 & 0.523599 & 0.498708 \\
\hline $\mathrm{A}_{2}$ & 0.569649 & 0.569476 & 0.632333 & 0.545937 & 0.579218 & 0.524008 \\
\hline $\mathrm{S}_{1}$ & 0.569141 & 0.547754 & 0.553558 & 0.5 & 0.527899 & 0.5 \\
\hline $\mathrm{S}_{2}$ & 0.523696 & 0.500000 & 0.523049 & 0.500000 & 0.500000 & 0.500000 \\
\hline W & 0.592464 & 0.626849 & 0.617619 & 0.629986 & 0.610936 & 0.609462 \\
\hline I & 0.498017 & 0.518059 & 0.519041 & 0.500000 & 0.500000 & 0.500000 \\
\hline $\mathrm{N}$ & 0.521807 & 0.500000 & 0.523049 & 0.500000 & 0.500000 & 0.524008 \\
\hline
\end{tabular}

\section{Discussion}

By using FCM technique and metacognition and communication data from teambased AAWC experiment, we are able to develop the cognitive mapping models in dynamic environment. It shows how the communication noise and metacognitive behavior (over or under confident) influence the different team selection (EI and II group). For example, according to the steady state results (Table 3), EI group shows that the identification task of the unknown aircrafts can be influenced by the unnecessary conversation between teammates $( \pm 0.012)$. However, it does not have influence on II group $( \pm 0)$. In addition, for EI group, the under-confident metacognitive behavior increases the frequencies of recognizing the Call ID $\left(\mathrm{R}_{1}\right)$ and reconfirming the identification of unknown aircrafts $\left(\mathrm{A}_{2}\right)$ as compared to the over-confident judgment condition $\left(\mathrm{R}_{1}:+0.06, \mathrm{~A}_{2}:+0.06\right)$. However, for II group, under-confident condition decreases frequencies of both $\mathrm{R}_{1}$ and $\mathrm{A}_{2}$ as compared to the over-confident metacognitive behavior $\left(\mathrm{R}_{1}:-0.03, \mathrm{~A}_{2}:-0.02\right)$. Our preliminary findings of this study show that FCM technique is useful to understand evolving metacognitive process in terms of the team performance and communication between teammates. The next step of the analysis is identifying the navigational training impact of the metacognitive behavior derived from team-communication. 


\section{References}

1. Kim, J.H., Macht, G.A., Li, S.: Comparison of Individual and Team-Based Dynamic Decision-Making Task (Anti-Air Warfare Coordinator): Consideration of Subjective Mental Workload Metacognition. In: Proceedings of the Human Factors and Ergonomics Society Annual Meeting. SAGE Publications (2012)

2. Kim, J., et al.: Investigating the effects of metacognition in dynamic control tasks. HumanComputer Interaction. Design and Development Approaches, 378-387 (2011)

3. Kosko, B.: Fuzzy cognitive maps. International Journal of Man-Machine Studies 24(1), 65-75 (1986)

4. Entin, E.E., Serfaty, D.: Adaptive team coordination. Human Factors: The Journal of the Human Factors and Ergonomics Society 41(2), 312-325 (1999)

5. Nelson, T.O., Narens, L.: Metamemory: A theoretical framework and new findings. The Psychology of Learning and Motivation 26, 125-141 (1990)

6. Sussan, D., Son, L.: The training of metacognitive monitoring in children. Columbia Undergraduate Science Journal 2(1) (2007)

7. Perry, N.E., Phillips, L., Hutchinson, L.R.: Preparing student teachers to support for selfregulated learning. Elementary School Journal 106(3), 237-254 (2006)

8. Zadeh, L.A.: Fuzzy sets. Information and Control 8(3), 338-353 (1965)

9. Ndousse, T., Okuda, T.: Computational intelligence for distributed fault management in networks using fuzzy cognitive maps. IEEE (1996)

10. Langan-Fox, J., Langfield-Smith, K.: Team mental models: Techniques, methods, and analytic approaches. Human Factors: The Journal of the Human Factors and Ergonomics Society, 2000 42(2), 242-271 (2000)

11. Xirogiannis, G., Stefanou, J., Glykas, M.: A fuzzy cognitive map approach to support urban design. Expert Systems with Applications 26(2), 257-268 (2004)

12. Jones, R.E.T., et al.: Modeling situation awareness for Army infantry platoon leaders using fuzzy cognitive mapping techniques (2010)

13. Dougherty, M.R., et al.: Using the past to predict the future. Memory \& Cognition 33(6), 1096-1115 (2005)

14. John, O.P., Naumann, L.P., Soto, C.J.: Paradigm shift to the integrative Big Five trait taxonomy. Handbook of Personality: Theory and Research, 114-158 (2008)

15. Garson, D.: Cluster Analysis (2008), http://www2 .chass.ncse.edu/garson/ PA765/cluster.htm

16. Barry, B., Stewart, G.L.: Composition, process, and performance in self-managed groups: The role of personality. Journal of Applied Psychology 82(1), 62 (1997)

17. Barrick, M.R., et al.: Relating member ability and personality to work-team processes and team effectiveness. Journal of Applied Psychology 83(3), 377 (1998)

18. Rothrock, L.: Using Time Windows to Evaluate Operator Performance. International Journal of Cognitive Ergonomics 5(1), 1-21 (2001)

19. Brannick, M.T., Roach, R.M., Salas, E.: Understanding team performance: A multimethod study. Human Performance 6(4), 287-308 (1993)

20. Adams, S.K.: Disciplinarily Hetero- and Homogeneous Design Team Convergence. Virginia Polytechnic Institute and State University, Blacksburg, VA. Doctoral Thesis (2007)

21. Letsky, M.P.: Macrocognition in teams: Theories and methodologies. Ashgate Publishing Company (2008)

22. Reimann, S.: On the design of artificial auto-associative neuronal networks. Neural Networks 11(4), 611-621 (1998) 